\title{
Fluctuation based proof of the stability of ac spectra of random operators on tree graphs
}

\author{
Michael Aizenman, Robert Sims, and Simone Warzel
}

\begin{abstract}
We summarize recent works on the stability under disorder of the absolutely continuous spectra of random operators on tree graphs. The cases covered include: Schrödinger operators with random potential, quantum graph operators for trees with randomized edge lengths, and radial quasi-periodic operators perturbed by random potentials.
\end{abstract}

\section{Introduction}

The incorporation of disorder is known to have strong effects on the spectral properties of self adjoint operators and the dynamics they generate. The phenomenon is relevant for models of physics, where it carries significant implications for the conduction properties of metals, the quantum hall effect, and the properties of quantum networks. As has been shown in various disciplines, disorder is a fruitful subject for mathematics. Yet, our understanding of its implications for random operators is still underdeveloped. This is exemplified by the fact that there is, at present, no example of a local operator in a finite dimensional space for which the existence of continuous spectrum has been established in the presence of weak but extensive, i.e., homogeneously random, disorder. Until recently, the one case for which there has been a constructive result, due to A. Klein K95, K98, is the discrete Schrödinger operator with an iid random potential on a regular Bethe lattice. Our recent work presents another method, with a different range of applicability, for the proof of the stability, under weak disorder, of ac spectra of tree operators. In this article we summarize the results and the main ideas which play a role in their derivation.

1.1. Examples of operators with disorder. A guiding example of a local generator of quantum dynamics is the Schrödinger operator

$$
H_{0}=-\Delta+U
$$

acting in $L^{2}\left(\mathbb{R}^{d}\right)$ with $\Delta$ the Laplacian and $U$ a potential which, for instance, could be periodic. Electric and magnetic fields can be taken into account as well, and in the latter case the operator loses its time reversal symmetry - the operator, and

1991 Mathematics Subject Classification. Primary 82B44; Secondary 47B80.

Key words and phrases. Random Schrödinger operators, ac spectrum, tree graphs.

The article covers talks given at UAB Int. Conf. on Diff. Eq. and Math. Phys., Birmingham, March 2005 (RS and SW), and at AMS Snowbird Conference, Utah, June 2005 (MA). 
its eigenfunctions, are no longer real. Disorder can be incorporated through the addition of a random potential, e.g.,

$$
V(\omega)=\sum_{\alpha \in \mathbb{Z}^{d}} \omega_{\alpha} u(\cdot-\alpha)
$$

with $\left\{\omega_{\alpha}\right\}_{\alpha}$ iid random variables and $u(\cdot)$ a local 'potential bump'. In this fashion one obtains a random operator:

$$
H_{\lambda}(\omega)=H_{0}+\lambda V(\omega) .
$$

The issues of interest can also be studied in the simpler context of the discrete analog of $H_{\lambda}(\omega)$ acting in $\ell^{2}(\mathbb{G})$, where $\mathbb{G}$ is a graph, e.g. $\mathbb{Z}^{d}$, and the random potential is described by a collection of random variables associated with the graph vertices.

Another example, which provides an interesting test-case for a number of concepts - effects of randomness, trace formulae, quantum wires - is the quantum graph operator $H_{0}=-d^{2} / d x^{2}$ acting on functions supported on a 'mesh of wires'. Accordingly, $H_{0}$ is defined in $\bigoplus_{e \in E} L^{2}\left(0, L_{e}\right)$, where $e$ ranges over the set of bonds of a graph, and $L_{e}$ is the length of the corresponding edge. It is made self-adjoint through a choice of boundary conditions at the graph vertices. A natural way to incorporate disorder is to vary the edge lengths, e.g., letting it be of the form

$$
L_{e}(\omega)=L_{0} e^{\lambda \omega_{e}}
$$

with $\left\{\omega_{e}\right\}$ iid random variables, as depicted in Figure 1 Another form is through random vertex rules, as in KoS99.

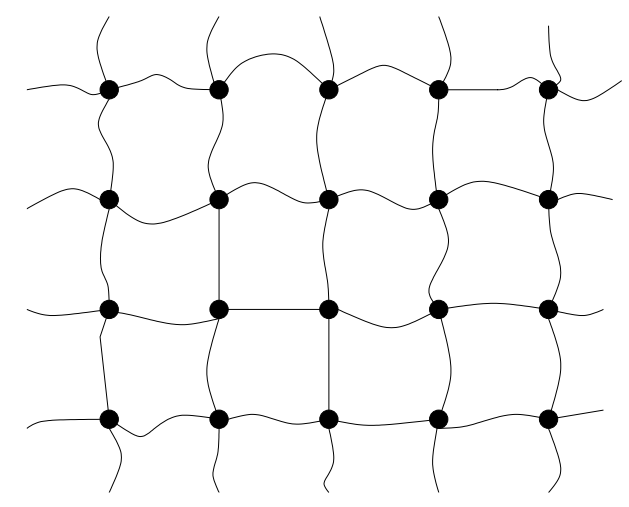

Figure 1. A mesh with random length on which the quantum graph operator $-d^{2} / d x^{2}$ may be defined.

The disorder is termed here extensive if its distribution is homogeneous. It is convenient to note that the distribution can be ergodic under the symmetry group of the (lattice) translations or graph isomorphism. This property guarantees KS80, P80, KM82 that the spectra and spectral components of such random operators are deterministic in the sense that there exist Borel sets $\Sigma, \Sigma_{\mathrm{ac}}, \Sigma_{\mathrm{pp}}, \Sigma_{\mathrm{sc}}$, such that with probability one, i.e., almost surely, the spectrum of $H(\omega)$ is given by $\Sigma$ :

$$
\sigma(H(\omega)) \stackrel{\text { a.s. }}{=} \Sigma
$$


and similarly for the pure-point, the absolutely continuous, and the singular-continuous components of the spectra.

1.2. Spectral regimes. The absolutely continuous (ac) component of the spectrum is of particular interest in condensed matter physics. In the basic 'electrongas' models of metals, the extended (generalized) eigenfunctions corresponding to the ac component play the main role in conduction. The last statement needs to be qualified. Certain conduction issues, such as adiabatic transport and the celebrated quantum hall effect, also require a good understanding of the implications of pure-point spectra H82, BSE94, ASS94, AG98. Moreover, in the absence of ac spectrum, the divergence of the localization length, even at a single energy, plays an important role in the dynamics generated by $H_{\lambda}(\omega)$ DSS02. The previous examples show that the relation of spectral and transport properties can be subtle. However, as was noted by Miller and Derrida MD93, the following simple hypothetical experiment illustrates the correspondence of ac spectra with conduction.

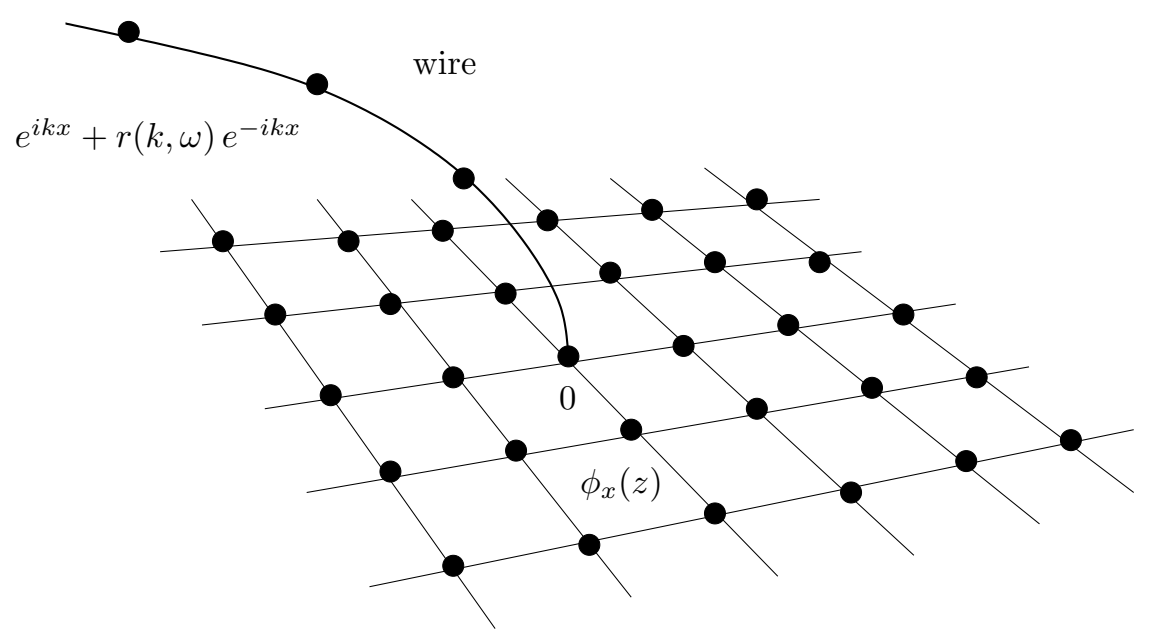

Figure 2. The setup for a scattering experiment. Current is sent down a wire which is attached to the graph.

Consider attaching an external wire at a vertex 0 and sending down it particles to probe the graph; as depicted in Figure 2. For simplicity, assume that the dynamics of the particle is generated by an operator which, within the graph, is given by $H_{\lambda}(\omega)$, along the wire is given by the one-dimensional operator $-\Delta+C$ with some constant potential $C$, and the contact is described through a suitable local rule. When particles are sent down at energy $E$ and decay rate $\eta>0$ the steady state amplitude for observing a particle at $x$ is given by

$$
\psi_{x}(z)= \begin{cases}e^{i k x}+r(k ; \omega) e^{-i k x} & \text { along the wire } \\ \phi_{x}(z) & \text { in the graph, }\end{cases}
$$

where $z=E+i \eta=4 \sin ^{2}(k / 2)+C$ and $\phi(z)$ is an $\ell^{2}$-solution of the Schrödinger equation $(-\Delta+\lambda V(\omega)-z) \phi=$ const. $\delta_{0}$ in the graph. This solution is unique up 
to a constant factor. Here $r(k ; \omega)$ is the reflection coefficient. Its value is readily determined from the resolvent, and one finds:

$$
|r(k ; \omega)|<1 \quad \Leftrightarrow \quad \operatorname{Im}\left\langle\delta_{0}, \frac{1}{H_{\lambda}(\omega)-E-i 0} \delta_{0}\right\rangle>0 .
$$

The graph absorbs the current of particles and conducts it to infinity if $|r(k ; \omega)|<1$, and we see that this happens precisely when there is an ac component in the spectral decomposition of $\delta_{0}$, as its density is given by the imaginary part of the diagonal element of the Green function times $\pi^{-1}$.

The remaining component of the spectral measure is referred to as the singular part. In a phenomenon known as Anderson localization An58, this part almost surely includes a pure-point component which consists of a countable collection of eigenvalues $\left\{E_{n}(\omega)\right\}$ forming a dense subset of the non-random set $\Sigma_{\mathrm{pp}}$. The corresponding eigenfunctions are exponentially localized, satisfying:

$$
\left|\psi_{n ; x}(\omega)\right| \leq A_{n}(\omega) e^{-\left|x-x_{n}(\omega)\right| / \xi} .
$$

Random Schrödinger operators have been the subject of intensive mathematical research CL90, PF92, St01. However, most of the results which were obtained pertain to the regime of Anderson localization, with the above characteristics.

Localization is expected to be more pronounced in low spatial dimensions. Indeed, among the first rigorous results was a proof [GMP77] that for a class of one dimensional random Schrödinger operators

$$
\sigma\left(H_{\lambda}(\omega)\right)=\Sigma_{\mathrm{pp}}
$$

at any strength of the disorder, $\lambda \neq 0$. Later works confirmed that (1.9) is generic for one dimensional Schrödinger operators with extensive disorder; for a review of results see St02. In all dimensions Anderson localization is present for large disorder or energies outside the spectrum of the zero-disorder counterpart of the operator. This has been established rigorously for various models using either the multiscale-approach [FS83, FMS85, DK89, GK01] or the fractional moment method AM93, A94, A+05. Aside from that, a common prediction is that part of the ac spectrum should be stable under weak disorder provided the spatial dimension is greater than two. So far, this question has been largely untouched by the rigorous works. The only existing results are for the "infinite dimensional" case of tree operators K95, K96, K98, ASW05a, ASW05b, AW05, as depicted in Figure 3.

The purpose of this short note is to summarize the essentials of ASW05a, ASW05b, AW05 where the tree scenario is revisited and a new method for establishing the stability of the ac spectrum for tree operators is presented. In particular, we will review the fluctuation based argument which is at the core of that method.

\section{Discrete Schrödinger operator on a regular rooted tree}

Let $\mathbb{T}$ be the set of vertices of a regular rooted tree with branching number $K \geq 2$. The tree version of the discrete random Schrödinger operator, acting in $\ell^{2}(\mathbb{T})$, is

$$
H_{\lambda}(\omega)=T+U+\lambda V(\omega)
$$




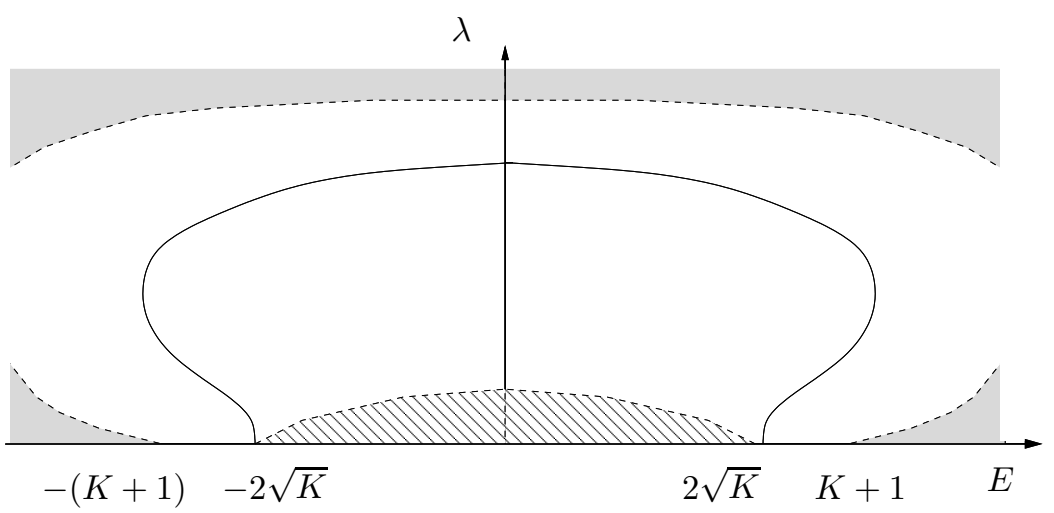

Figure 3. A schematic sketch of the 'phase diagram' of the spectrum of the discrete Schrödinger operator on a homogeneous tree, $H_{\lambda}(\omega)=T+\lambda V(\omega)$ with iid random potential of zero mean. The solid line indicates the expected mobility edge separating the ac and the pure-point spectral regimes. The dotted lines delineate regions for which ac (lined) and pure point (shaded) spectra were established. It is conjectured that finite dimensional graphs have a similar phase diagram, however there is at present no proof of the existence of ac spectrum in any example of a local operator with homogeneous disorder in finite dimensions.

where $T$ is the adjacency matrix, $U$ is a potential which is radial and periodic in the distance to the root, and $V(\omega)$ a random potential given by a sequence $\omega=\left\{\omega_{x}\right\}_{x \in \mathbb{T}}$ of random variables. The parameter $\lambda \in \mathbb{R}$ controls here the strength of the disorder. This version of the discrete random Schrödinger operator has attracted attention early on, as an instructive example for the study of Anderson localization [AAT, AT74.

Let us first note what is known about the spectral properties of the background operator corresponding to $\lambda=0$. Here the following observation, which we take from ASW05a, is useful.

Lemma 2.1. If $\left\{V_{x}\right\}_{x \in \mathbb{T}}$ is a radial function on $\mathbb{T}$, then the ac spectrum of $H=T+V$ on $\ell^{2}(\mathbb{T})$ coincides with the scaled up version of the ac spectrum of $h^{+}=T+V / \sqrt{K}$ on $\ell^{2}\left(\mathbb{N}_{0}\right)$, satisfying:

$$
\sigma_{\mathrm{ac}}(H)=\sqrt{K} \sigma_{\mathrm{ac}}\left(h^{+}\right) .
$$

For the singular spectrum an inclusion holds, $\sigma_{\text {sing }}(H) \supset \sqrt{K} \sigma_{\text {sing }}\left(h^{+}\right)$.

This statement has the following consequences for radial tree operators:

i. The ac spectrum of $H_{0}=T+U$ on $\ell^{2}(\mathbb{T})$ is non-empty, $\sigma_{\mathrm{ac}}\left(H_{0}\right) \neq \emptyset$, and, as with periodic potentials, has a band structure. An example is $\sigma_{\mathrm{ac}}(T)=[-2 \sqrt{K}, 2 \sqrt{K}]$, in which case there are no gaps.

ii. The ac spectrum is unstable under perturbations by arbitrarily weak but radially symmetric disorder of homogeneous strength. For the random operator defined by setting $\omega_{x}=\xi_{|x|}$, with $\left\{\xi_{n}\right\}_{n \in \mathbb{N}_{0}}$ a sequence of iid 
non-constant random variables, at any $\lambda \neq 0$ :

$$
\sigma_{\mathrm{ac}}\left(H_{\lambda}(\omega)\right)=\emptyset
$$

for almost every $\omega$. This is due to the well known effect of disorder in one dimension.

The last comment is to be contrasted with the main result presented below. As will be explained, it shows that the ac spectra are stable under weak disorder which is either uncorrelated, or meets the following weak-correlation condition.

Definition 2.2. The random variables $\left\{\omega_{x}\right\}_{x \in \mathbb{T}}$ are said to be weakly correlated iff there exists a $\kappa \in(0,1]$ such that for any $x \neq y \in \mathbb{T}$ which are common forward

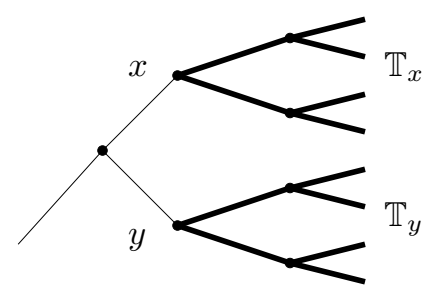

neighbors to some vertex and any pair of functions $X, Y: \mathbb{R}^{\mathbb{T}} \rightarrow[0, \infty)$ measurable with respect to the $\sigma$-algebra associated with the forward subtrees $\mathbb{T}_{x}$ and $\mathbb{T}_{y}$ respectively, one has

$$
\mathbb{E}[X(\omega) Y(\omega)] \geq \kappa \mathbb{E}[X(\omega)] \mathbb{E}[Y(\omega)] .
$$

Any iid sequence forms a trivial example of such weakly correlated random variables. A broader class of examples can be found in ASW05a.

The main result of ASW05a reads as follows:

THEOREM 2.3. Let $\left\{\omega_{x}\right\}_{x \in \mathbb{T}}$ be stationary, weakly correlated, and suppose that $\mathbb{E}\left[\log \left(1+\left|\omega_{0}\right|\right)\right]<\infty$. Then, for every $I \subset \sigma_{\mathrm{ac}}\left(H_{0}\right)$ with $I \cap \sigma_{\operatorname{sing}}\left(H_{0}\right)=\emptyset$ :

$$
\begin{gathered}
\lim _{\lambda \rightarrow 0} \mathbb{E}\left[\int_{I}\left|\operatorname{Im}\left\langle\delta_{0}, \frac{1}{H_{\lambda}(\omega)-E-i 0} \delta_{0}\right\rangle-\operatorname{Im}\left\langle\delta_{0}, \frac{1}{H_{0}-E-i 0} \delta_{0}\right\rangle\right| d E\right] \\
=0,
\end{gathered}
$$

Some remarks are in order:

i. Eq. 2.5 is a statement of $L^{1}$-convergence of the ac densities, which of course implies weak convergence of the ac components of the spectral measures. As a consequence of (2.5) the ac spectrum is stable in Lebesgue sense, i.e., for all Borel sets $I \subset \mathbb{R}$

$$
\lim _{\lambda \rightarrow 0}\left|\sigma_{\mathrm{ac}}\left(H_{\lambda}(\omega)\right) \cap I\right|=\left|\sigma_{\mathrm{ac}}\left(H_{0}\right) \cap I\right|,
$$

where $|\cdot|$ denotes the Lebesgue measure.

ii. In the case of $U=0$ and $\left\{\omega_{x}\right\}_{x \in \mathbb{T}}$ iid, more is known. Klein K95, K98 proved that for every $0<|E|<2 \sqrt{K}$ there exists $\lambda(E)>0$ such that

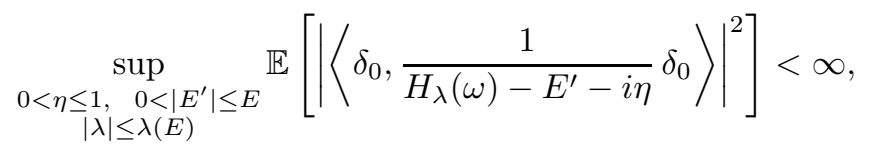


which is sufficient to ensure that the spectrum in this interval is purely absolutely continuous.

iii. For the last example $(U=0)$ pure point spectrum was proven at large disorder and also for small disorder and energies $|E| \geq K+1$ in [A94. This leaves still unresolved the issue of band edge localization in the energy regime $|E| \in[2 \sqrt{K}, K+1]$.

\section{Radial quasi-periodic operators}

Theorem 2.3 has been extended in AW05 to certain cases where $\left\{U_{x}\right\}_{x \in \mathbb{T}}$ is a radial and quasi-periodic (qp) function, i.e.,

$$
U_{x}(\theta)=u\left(S^{|x|} \theta\right),
$$

where $u: \Xi \rightarrow \mathbb{R}$ is a continuous function on a multidimensional torus $\Xi$ on which $S$ acts as an ergodic shift, $S \theta=(\theta+2 \pi \alpha) \bmod 2 \pi$, with a frequency vector $\alpha$. The stability results then refers to the operator

$$
H_{\lambda}(\theta, \omega)=T+U(\theta)+\lambda V(\omega)
$$

on $\ell^{2}(\mathbb{T})$. The operator $H_{0}(\theta)$ represents a 'fanned out' version of the qp onedimensional operator

$$
h(\theta)=T+u\left(S^{|x|} \theta\right) / \sqrt{K}
$$

on $\ell^{2}(\mathbb{Z})$. The precise assumptions on $u$ are formulated in terms of the extensively studied properties of $h(\theta)$.

For a variety of one-dimensional qp operators $h(\theta)$ it has been established by KAM and duality methods that they admit pairs of Bloch-Floquet eigenstates for almost all energies in its ac spectrum DS75, BLT83, Si87, CD89, E92, Pu04, G+97, J99, BJ02, Pu05. The latter are defined as follows.

Definition 3.1. The operator $h(\theta)$ is said to admit a Bloch-Floquet eigenstate with energy $E$ and quasi-momentum $k \in(-\pi, \pi]$ iff there is a non-vanishing function $\psi: \mathbb{Z} \times \Xi \rightarrow \mathbb{C}$ such that

(i) $h(\theta) \psi(\theta)=E \psi(\theta)$

in the weak sense

(ii) $\psi_{x}(\theta)=\exp (i k x) \varphi\left(S^{x} \theta\right)$

for some continuous $\varphi: \Xi \rightarrow \mathbb{C}$.

Bloch-Floquet eigenstates can be viewed as states which are covariant functions on the operator, with respect to translations. They naturally come in pairs: if $\psi(\theta)$ is one, then its complex conjugate is also one with the same energy, but reversed quasi-momentum. While not true for all [L93, many qp operators $h(\theta)$ admit pairs of linearly independent, complex-conjugate Bloch-Floquet eigenstates throughout their ac spectra (with the exclusion of countable collections of energies). The most prominent example is the almost Mathieu operator which corresponds to $u(\theta)=u_{0} \cos (\theta)$ with $\theta \in[0,2 \pi)$.

Within this setup, the main result of AW05 is:

Theorem 3.2. Let $\left\{\omega_{x}\right\}_{x \in \mathbb{T}}$ be as in Theorem 2.3 Suppose that for almost every $E \in \sigma_{\mathrm{ac}}(h(\theta))$ the operator $h(\theta)$ admits a pair of linearly independent, complexconjugate Bloch-Floquet eigenstates. Then, in the weak disorder limit:

$$
\lim _{\lambda \rightarrow 0}\left|\sigma_{\mathrm{ac}}\left(H_{\lambda}(\theta, \omega)\right) \cap I\right|=\left|\sigma_{\mathrm{ac}}\left(H_{0}(\theta)\right) \cap I\right| .
$$


The rôle played by the Bloch-Floquet eigenstates in the proof will be briefly commented upon at the end of Section 5

\section{Random quantum trees}

In this section we consider the Laplacian on the Hilbert space $\bigoplus_{e \in E} L^{2}\left(0, L_{e}\right)$ where $E$ is the set of edges corresponding to $\mathbb{T}$. For any edge $e \in E$ the length of $e$ is denoted by $L_{e}$ with orientation (in particular, the direction of increase) taken away from the root. Any function $\psi$ in the above Hilbert space may be written as $\psi=\left\{\psi_{e}\right\}_{e \in E}$, and the quantum tree graph Laplacian acts as a second derivative

$$
\Delta_{\mathbb{T}} \psi=\left\{\psi_{e}^{\prime \prime}\right\}_{e \in E}
$$

on the domain of functions $\psi$ for which each $\psi_{e} \in H^{2}\left(0, L_{e}\right)$ subject to certain boundary conditions, which we take as follows:

i. at the root:

$$
\cos (\alpha) \psi(0)-\sin (\alpha) \psi^{\prime}(0)=0,
$$

for some fixed $\alpha \in[0, \pi)$.

ii. at internal vertices the functions are required to satisfy the Kirchoff/Neumann conditions, i.e., $\psi$ is continuous and

$$
\psi_{e}^{\prime}\left(L_{e}\right)=\sum_{e^{\prime} \in \mathcal{N}_{e}^{+}} \psi_{e}^{\prime}(0) .
$$

These conditions guarantee that $\Delta_{\mathbb{T}}$ is self-adjoint $\mathbf{K S 9 9}$ and that $\sigma_{\mathrm{ac}}\left(-\Delta_{\mathbb{T}}\right)$ is independent of $\alpha$.

For the regular tree, i.e., $L_{e}=L$ for all $e \in E$, the spectrum is explicitly known SoS02, So04,

$$
\sigma_{\mathrm{ac}}\left(-\Delta_{\mathbb{T}}\right)=\bigcup_{n=0}^{\infty}\left[\left(\frac{\pi(n+1)+\theta}{L}\right)^{2},\left(\frac{\pi n+\theta}{L}\right)^{2}\right],
$$

where $\theta=\arctan \frac{K-1}{2 \sqrt{K}}$. Moreover, there occur infinitely degenerate eigenvalues in the band gaps at the Dirichlet eigenvalues of the Laplacian on an interval of length $L$.

Our aim is to study random deformations $\mathbb{T}_{\lambda}(\omega)$ of such a regular tree graph. We keep the vertex set fixed but define random edge lengths in terms of

$$
L_{e}(\omega):=L \exp \left(\lambda \omega_{e}\right)
$$

where $\omega=\left\{\omega_{e}\right\}_{e \in E}$ are bounded iid random variables, and $\lambda \in[0,1]$ is the disorder parameter. For this setup the main result of ASW05a reads as follows.

Theorem 4.1. For any Borel set $I \subset \mathbb{R}$ and almost every $\omega$,

$$
\lim _{\lambda \rightarrow 0}\left|\sigma_{\mathrm{ac}}\left(-\Delta_{\mathbb{T}_{\lambda}(\omega)}\right) \cap I\right|=\left|\sigma_{\mathrm{ac}}\left(-\Delta_{\mathbb{T}}\right) \cap I\right| \text {. }
$$

Let us close this section with few remarks:

i. The result of Theorem 4.1 remains true if $\left\{\omega_{e}\right\}_{e \in E}$ are weakly correlated and it is expected to be false if $\left\{\omega_{e}\right\}_{e \in E}$ is radial. The results are also valid if one replaces (4.3) by some $\alpha$-type boundary conditions - provided $\alpha$ is either constant or a radial function of $x$. 
ii. There are only a few published results on localization for quantum graphs. These are generally expected to be similar to those of the discrete Schrödinger operators. However, this setup provides a natural context for the study of other issues. The work ScS00 presents a localization result for one-dimensional quantum graphs derived through the analysis of the interference of periodic orbits in the presence of random scatterers. Better understanding of such cancellations is of independent interest with relation to trace formulae and issues of dynamics in the presence of disorder.

\section{Sketch of the proof}

In the remainder, we will sketch the proof of the stability of the ac spectrum for the random tree models which were introduced above. For simplicity, we focus on the discrete model (2.1) with $U=0$. As is shown in ASW05b, a similar analysis applies to the quantum graph model, once one has identified the appropriate quantities.

Our analysis begins by identifying a particular solution, familiar from the study of one dimensional Sturm-Liouville equations, which we refer to as the WeylTitchmarsh function. This function can be understood from a scattering theoretic perspective, and within this context, one is naturally led to certain observations concerning the conservation (or loss, when $\eta \neq 0$ ) of current along the edges of the tree. Analysis of the current conservation inequality provides a bound on the fluctuations of the random Green functions in terms of a quantity which we describe as a Lyapunov exponent on the tree. Similar to the one dimensional theory, this Lyapunov exponent, which is the negative of the real part of a Herglotz function, vanishes on the ac spectrum of the background operator. Quite generally, based on arguments which are natural for functions in $H^{p}$ spaces, averages of such quantities are continuous in their parameters, and from this fact, in particular, (5.13), we can prove that the distribution of these random Green's functions collapse to a unique point in the limit of weak disorder. Uniqueness of the solution of the underlying recursion relation identifies this point as the free Green's function. Theorem 2.3 follows from these observations, although some attention to the details is necessary.

5.1. The Weyl-Titchmarsh function on the tree. Consider the scattering experiment depicted in Figure 2 in case $\mathbb{Z}^{d}$ replaced by $\mathbb{T}$ and the wire is attached to the root of $\mathbb{T}$. Let $\psi(z)$ be an $\ell^{2}$-solution of the Schrödinger inside the tree, which

is unique up to a constant factor and vanishes nowhere. Therefore the following quantity, which we refer to as the Weyl-Titchmarch function, is unique and well defined.

Definition 5.1. For any $x \in \mathbb{T}$ and $z \in \mathbb{C}$ take

$$
\Gamma_{x}(z):=-\frac{\psi_{x}(z)}{\psi_{x^{-}}(z)}
$$

where $x^{-}$is the backward neighbor of $x$.

The function $\Gamma_{x}$ has several interesting properties:

i. Since $\psi(z)$ satisfies the Schrödinger equation, $\Gamma(z)$ satisfies the following recursion relation

$$
\Gamma_{x}(z)=\frac{1}{\lambda \omega_{x}-z-\sum_{y \in \mathcal{N}_{x}^{+}} \Gamma_{y}(z)},
$$


where $\mathcal{N}_{x}^{+}$is the set of forward neighbors of $x$.

ii. One may rewrite $\Gamma_{x}(z)$ in terms of the resolvent of $H_{\lambda}(\omega)$ restricted to the Hilbert space over the forward subtree $\mathbb{T}_{x}$ starting at $x$,

$$
\Gamma_{x}(z)=\left\langle\delta_{x}, \frac{1}{\left.H_{\lambda}(\omega)\right|_{\mathbb{T}_{x}}-z} \delta_{x}\right\rangle .
$$

Therefore, $\Gamma_{x}$ is a Herglotz-Nevanlinna function, i.e., $\Gamma_{x}$ is analytic in $\mathbb{C}^{+}$ and $\operatorname{Im} \Gamma_{x}(z)>0$ if $\operatorname{Im} z>0$.

iii. The ac-density of the Schrödinger operator $H_{\lambda}(\omega)$ associated with the vector $\delta_{0}$ at the root is given by $\operatorname{Im} \Gamma_{0}(E+i 0) / \pi$.

iv. The current carried by $\psi(z)$ along the bond from $x$ to $y$ is

$$
J_{x y}(z)=\left|\psi_{x}(z)\right|^{2} \operatorname{Im} \Gamma_{y}(z)
$$

and therefore the current conservation / loss at each vertex may be written as

$$
J_{x^{-} x}(z) \geq \sum_{y \in \mathcal{N}_{x}^{+}} J_{x y}(z)
$$

with equality for real $z$.

Rewriting (5.5) in terms of (5.4), taking the logarithm, and then averaging yields

$$
\mathbb{E}\left[\log \frac{1}{K} \sum_{y \in \mathcal{N}_{x}^{+}} \operatorname{Im} \Gamma_{y}\right]-\mathbb{E}\left[\log \operatorname{Im} \Gamma_{x}\right] \leq \mathbb{E}\left[\log \frac{1}{K}\left|\frac{\psi_{x-}}{\psi_{x}}\right|^{2}\right]=: 2 \gamma_{\lambda}
$$

The quantity right side of (5.6) may be identified as twice a Lyapunov exponent on the tree, since it measures the average decay rate of $\psi$ in one generation. A standard application of Jensen's inequality to the left side of (5.6) demonstrates that this quantity is non-negative. As the Jensen inequality is strict unless the random variables (over which one averages) cease to fluctuate, we are naturally led to the core of our argument, which will be presented in the next subsection.

5.2. Fluctuation Bounds. We will bound the fluctuations of the random variables $\Gamma_{x}$ in terms of the Lyapunov exponent introduced above. To this end, we require an improvement of the Jensen inequality which quantifies the 'boost' due to fluctuations.

Lemma 5.2. Let $\left\{X_{j}\right\}_{j=1}^{K}$ with $K \geq 2$ be positive, weakly correlated, identically distributed random variables. Then, for any $\alpha \in(0,1 / 2]$,

$$
\mathbb{E}\left[\log \frac{1}{K} \sum_{j=1}^{K} X_{j}\right] \geq \mathbb{E}\left[\log X_{1}\right]+\frac{\alpha^{2} \kappa}{4} \delta\left(X_{1}, \alpha\right)^{2},
$$

with the relative $\alpha$-width of $X_{1}$ which is defined as

$$
\delta\left(X_{1}, \alpha\right):=\frac{\xi_{+}\left(X_{1}, \alpha\right)-\xi_{-}\left(X_{1}, \alpha\right)}{\xi_{+}\left(X_{1}, \alpha\right)},
$$

where $\xi_{-}\left(X_{1}, \alpha\right):=\sup \left\{\xi: \mathbb{P}\left(X_{1}<\xi\right) \leq \alpha\right\}$ and $\xi_{+}\left(X_{1}, \alpha\right):=\inf \left\{\xi: \mathbb{P}\left(X_{1}>\right.\right.$ $\xi) \geq \alpha\}$.

Applying this result with $\left\{X_{j}\right\}_{j=1}^{K}$ taken to be $\left\{\operatorname{Im} \Gamma_{y}\right\}_{y \in \mathcal{N}_{x}^{+}}$, we obtain from (5.6) the first claim in the following 
Theorem 5.3. For all $\lambda \in \mathbb{R}, z \in \mathbb{C}^{+}$, and $\alpha \in(0,1 / 2]$ :

$$
\begin{aligned}
\delta\left(\operatorname{Im} \Gamma_{0}(z), \alpha\right)^{2} & \leq \frac{8}{\kappa \alpha^{2}} \gamma_{\lambda}(z), \\
\delta\left(\left|\Gamma_{0}(z)\right|^{2}, \alpha\right)^{2} & \leq \frac{32(K+1)^{2}}{\kappa \alpha^{2}} \gamma_{\lambda}(z) .
\end{aligned}
$$

Since we have bounded the fluctuations of $\Gamma_{x}$ in terms of the Lyapunov exponent, it is important to note several of its properties.

i. From (5.6), (5.1) and stationarity, we conclude that

$$
\gamma_{\lambda}(z)=-\mathbb{E}\left[\log \sqrt{K}\left|\Gamma_{0}(z)\right|\right] .
$$

Hence, $\gamma_{\lambda}$ is a non-negative harmonic function of $z \in \mathbb{C}^{+}$.

ii. Moreover, by Kotani theory Ko85 and the relation (2.2) of the ac spectrum of tree operators with radial potentials with that of the corresponding operator on the line, we have $\gamma_{0}(E+i 0)=0$ for almost every $E \in \sigma_{\mathrm{ac}}\left(H_{0}\right)$.

iii. For $\operatorname{Im} z>0, \gamma_{\lambda}(z)$ is jointly continuous in it's relevant parameters. This yields the following continuity statement, which is derived through the deformation of the contour of integration and the natural bounds on $\Gamma(z)$ :

$$
\lim _{\substack{\lambda \rightarrow 0 \\ \eta \rightarrow 0^{+}}} \int_{I} \gamma_{\lambda}(E+i \eta) d E=\int_{I} \gamma_{0}(E+i 0) d E=0,
$$

for any Borel set $I \subset \sigma_{\mathrm{ac}}\left(H_{0}\right)$.

The last relation means that, at least upon energy averaging, the right sides in (5.9) and (5.10) vanish in any joint limit $\lambda, \eta \rightarrow 0$. Accordingly, the relative fluctuations of $\operatorname{Im} \Gamma_{0}$ and $\left|\Gamma_{0}\right|$ vanish in this limit.

5.3. Putting it together. To complete the result, we consider all possible distributional limits of $\left\{\Gamma_{x}\right\}_{x \in \mathbb{T}}$ as $\lambda, \eta \rightarrow 0$, where the distribution $\mathcal{D}$ refers to jointly the energy in some Borel subset of $\sigma_{\mathrm{ac}}\left(H_{0}\right)$ and the randomness. It should be understood there that $\Gamma_{x}$ initially depends on $\{\omega, \lambda, \eta, E\}$, and we are considering the possible limits of these functions, for $\lambda, \eta \rightarrow 0$. All such limits, or accumulation points, are characterized by

i. the vanishing distributional width of $\operatorname{Im} \Gamma_{x}$ and $\left|\Gamma_{x}\right|$.

ii. the stationarity: $\Gamma_{x} \stackrel{\mathcal{D}}{=} \Gamma_{y}$

iii. the limiting recursion relation

$$
\Gamma_{x} \stackrel{\mathcal{D}}{=} \frac{1}{-E-\sum_{y \in \mathcal{N}_{x}^{+}} \Gamma_{y}},
$$

Since the (non-random) recursion relation $\Gamma=\frac{1}{-E-K \Gamma}$ has a unique fixed point in $\mathbb{C}^{+}$, we conclude the distributional convergence

$$
\Gamma_{x}(E+i \eta) \stackrel{\mathcal{D}}{\rightarrow} \Gamma_{x}(E+i 0),
$$

as $\lambda, \eta \rightarrow 0$. Outside the singular spectrum $\sigma_{\text {sing }}\left(H_{0}\right)$ this implies the $L^{1}$-convergence claimed in 2.5. 
In case $H_{0}$ is radially periodic, with period $\tau$, the stationarity of $\Gamma_{x}$ is replaced by periodicity, and in the recursion relation (5.14) the Möbius transformation is replaced by its $\tau$-iterate.

Let us finish with a remark on the issues showing up in this last step of the proof in case $U$ is quasi-periodic as given by (3.1) Here the above argument requires the uniqueness of solutions $\Gamma$ with values in the upper half plane of the cocycle equation

$$
\Gamma(\theta)=\frac{1}{u(\theta)-E-K \Gamma(\theta)}
$$

for almost all energies $E \in \sigma_{\mathrm{ac}}\left(H_{0}(\theta)\right)$. As was shown in [AW05, this is the case if $h(\theta)$ (given by (3.3) ) admits Bloch-Floquet eigenfunctions at Lebesgue-almost every energy in its ac spectrum. The argument relies on a result in Pu05 which proves the reducibility of the Schrödinger cocycle to a constant one under this assumption.

\section{Some open challenges}

It will be of interest to see progress on open issues related to the above discussion:

i. Extend the analysis of stability of the ac spectra to finite dimensions.

ii. Clarify a possible relation of the spectral type with the level statistics.

The latter refers to the distribution of the energy gaps, resolved on the scale of the mean energy spacing, for finite-volume versions of the corresponding operators. Some partial results on the last point will be described elsewhere.

\section{Acknowledgements}

The work described here has benefitted from our enjoyable and stimulating discussions with Thomas Chen at Princeton Univ. and Uzy Smilansky at Weizmann Inst. Sci., where MA has enjoyed kind hospitality at the Einstein Center for Theoretical Physics. The work was supported in parts by the NSF grant PHY-9971149 (MA), an NSF Postdoctoral Fellowship (RS), and by the DFG grant Wa 1699/1 $(\mathrm{SW})$.

\section{References}

[AAT] R. Abou-Chacra, P. W. Anderson, and D. J. Thouless. A selfconsistent theory of localization. J. Phys. C: Solid State Phys., 6:1734-1752, 1973.

[AT74] R. Abou-Chacra and D. J. Thouless. Self-consistent theory of localization. II. localization near the band edges. J. Phys. C: Solid State Phys., 7:65-75, 1974.

[AM93] M. Aizenman and S. Molchanov. Localization at large disorder and at extreme energies: an elementary derivation. Commun. Math. Phys., 157:245, 1993.

[A94] M. Aizenman. Localization at weak disorder: some elementary bounds. Rev. Math. Phys., 6:1163-1182, 1994.

[AG98] M. Aizenman and G. M. Graf. Localization bounds for an electron gas. J. Phys. A: Math. Gen., 31:6783-6806, 1998.

[A+05] M. Aizenman, A. Elgart, S. Naboko, J. H. Schenker, and G. Stolz, Moment analysis for localization in random Schrödinger operators. preprint math-ph/0308023 To appear in Invent. Math.

[ASW05a] M. Aizenman, R. Sims and S. Warzel. Stability of the absolutely continuous spectrum of random Schrödinger operators on tree graphs. Preprint math-ph/0502006 To appear in Prob. Theor. Rel. Fields.

[ASW05b] M. Aizenman, R. Sims and S. Warzel. Absolutely continuous spectra of quantum tree graphs with weak disorder. Preprint math-ph/0504039 To appear in Comm. Math. Phys. 
[AW05] M. Aizenman and S. Warzel. Persistence under weak disorder of AC spectra of quasiperiodic Schrödinger operators on tree graphs. Preprint math-ph/0504084 To appear in Mosc. Math. J.

[An58] P. W. Anderson. Absence of diffusion in certain random lattices. Phys. Rev., 109:1492-1505, 1958.

[ASS94] J. E. Avron, R. Seiler, and B. Simon. Charge deficiency, charge transport and comparison of dimensions. Comm. Math. Phys., 159:399-422, 1994.

[BLT83] J. Bellissard, R. Lima and D. Testard. A metal-insulator transition for the almost Mathieu model. Commun. Math. Phys. 88:207-234, 1983.

[BSE94] J. Bellissard, H. Schulz-Baldes, and A. van Elst. The non commutative geometry of the Quantum Hall Effect. J. Math. Phys. 35:5373-5471, 1994.

[BJ02] J. Bourgain and S. Jitomirskaya. Absolutely continuous spectrum for $1 D$ quasiperiodic operators. Invent. Math. 148:453-463, 2002.

[CL90] R. Carmona and J. Lacroix. Spectral theory of random Schrödinger operators. Birkhäuser, Boston, 1990.

[CD89] V. Chulaevsky and F. Delyon. Purely absolutely continuous spectrum for almost Mathieu operators. J. Stat. Phys. 55:1279-1284, 1989.

[DSS02] D. Damanik, R. Sims, and G. Stolz. Localization for one-dimensional, continuum, Bernoulli-Anderson models. Duke Math. J., 114:59-100, 2002.

[DS75] E. Dinaburg and Y. Sinai. The one-dimensional Schrödinger equation with quasiperiodic potential. Funkt. Anal. i. Priloz. 9:8-21, 1995.

[DK89] H. von Dreifus, and A. Klein. A new proof of localization in the Anderson tight binding model. Commun. Math. Phys., 124: 285-299, 1989.

[E92] L. H. Eliasson. Floquet slutions for the 1-dimensional quasiperiodic Schrödinger equation. Commun. Math. Phys. 146:447-482, 1992.

[FMS85] J. Fröhlich, F. Martinelli, E. Scoppola, and T. Spencer. Constructive proof of localization in the Anderson tight binding model. Commun. Math. Phys., 101:21-46, 1985.

[FS83] J. Fröhlich, and T. Spencer, Absence of diffusion in the Anderson tight binding model for large disorder or low energy. Commun. Math. Phys., 88:151-184, 1983.

[GK01] F. Germinet, and A. Klein. Bootstrap multiscale analysis and localization in random media. Commun. Math. Phys., 222: 415-448, 2001.

[GMP77] I. Ya. Goldsheid, S. Molchanov, and L. Pastur. A pure point spectrum of the stochastic one-dimensional Schrödinger operator. Funct. Anal. Appl., 11:1-8, 1977.

$[\mathrm{G}+97] \quad$ A. Y. Gordon, S. Jitomirskaya, Y. Last and B. Simon. Duality and singular continuous spectrum in the almost Mathieu equation. Acta Math. 178:169-183, 1997.

[H82] B. I. Halperin. Quantized Hall conductance, current-carrying edge states, and the existence of extended states in a two-dimensional disordered potential. Phys. Rev. B $25: 2185-2190,1982$.

[J99] S. Jitomirskaya. Metal-insulator transition for the almost Mathieu operator. Ann. Math. 150:1159-1175, 1999.

[KM82] W. Kirsch, and F. Martinelli. On the ergodic properties of the spectrum of general random operators. J. Reine Angew. Math., 334:141-156, 1982.

[K95] A. Klein. The Anderson metal-insulator transition on the Bethe lattice. In D. Iagolnitzer, editor, Proceedings of the XIth international congress on mathematical physics, Paris, France, July 18-23, 1994, pages 383-391. International Press, Cambridge, MA, 1995.

[K96] A. Klein. Spreading of wave packets in the Anderson model on the Bethe lattice. Commun. Math. Phys., 177:755-773, 1996.

[K98] A. Klein. Extended states in the Anderson model on the Bethe lattice. Adv. Math., 133:163-184, 1998.

[KS99] V. Kostrykin and R. Schrader. Kirchhoff's rule for quantum wires. J. Phys. A 32:595630, 1999.

[Ko85] S. Kotani. One-dimensional random Schrödinger operators and Herglotz functions. In K. Ito, editor, Taneguchi Symp. PMMP, pages 219-250, Amsterdam, 1985. North Holland.

[KoS99] T. Kottos and U. Smilansky. Periodic orbit theory and spectral statistics for quantum graphs. Ann. Phys. 274:76-124, 1999. 
[KS80] H. Kunz and B. Souillard. Sur le spectre des opérateurs aux différence finies aléatoires. Commun. Math. Phys., 78:201-246, 1980.

[Ku02] P. Kuchment. Graph models for waves in thin structures. Waves and random media, 12:R1-R24, 2002.

[Ku04] P. Kuchment. Quantum graphs: I. Some basic structures. Waves and random media, 14:S107-S128, 2004.

[Ku05] P. Kuchment. Quantum graphs II. Some spectral properties of quantum and combinatorial graphs, J. Phys. A: Math. Gen. 38:4887-4900 (2005).

[L93] Y. Last. A relation between a.c. spectrum of ergodic Jacobi matrices and the spectra of periodic approximants. Commun. Math. Phys. 151:183-192, 1993.

[MD93] J. D. Miller and B. Derrida. Weak disorder expansion for the Anderson model on a tree. J. Stat. Phys, 75:357-388, 1993.

[P80] L. A. Pastur. Spectral properties of disordered systems in the one body approximation. Commun. Math. Phys., 75:167-196, 1980.

[PF92] L. Pastur and A. Figotin. Spectra of random and almost-periodic operators. SpringerVerlag, Berlin, 1992.

[Pu04] J. Puig. Cantor spectrum for the almost Mathieu operator. Commun. Math. Phys. 244:297-309, 2004.

[Pu05] J. Puig. A non-perturbative Eliasson's reducibility theorem. Preprint math.DS/0503356

[ScS00] H. Schanz and U. Smilansky. Periodic-orbit theory of Anderson-localization on graphs. Phys. Rev. Lett. 84:1427-1430, 2000.

[S83] B. Simon. Kotani theory for one-dimensional Jacobi matrices. Commun. Math. Phys., 89:227-234, 1983.

[Si87] Y. G. Sinai. Anderson localization for one-dimensional difference Schrödinger operator with quasiperiodic potential. J. Stat. Phys. 46:861-909, 1987.

[SoS02] A. V. Sobolev and M. Solomyak. Schrödinger operators on homogeneous metric trees: spactrum in gaps. Rev. Math. Phys., 14: 421-467, 2002.

[So04] M. Solomyak. On the spectrum of the Laplacian on regular metric trees. Waves and random media, 14:S155-S171, 2004.

[St01] P. Stollmann. Caught by disorder: bound states in random media. Birkhäuser, Boston, 2001.

[St02] G. Stolz. Strategies in localization proofs for one-dimensional random Schrödinger operators. Proc. Indian Acad. Sci., Math. Sci. 112:229-243, 2002.

Departments of Mathematics and Physics, Princeton University, Princeton NJ 08544, USA

E-mail address: aizenman@princeton.edu

Department of Mathematics, University of California at Davis, Davis CA 95616, USA

E-mail address: rjsims@math.ucdavis.edu

Department of Mathematics, Princeton University, Princeton NJ 08544, USA

E-mail address: swarzel@princeton.edu 